\title{
A portable positron accumulator for antihydrogen formation
}

\author{
R.S. Conti, B. Ghaffari and T.D. Steiger \\ The University of Michigan, Ann Arbor, MI48109, USA
}

\begin{abstract}
A pulsed source of positrons has been developed which may be useful for antihydrogen $(\overline{\mathrm{H}})$ formation because it is portable when compared to accelerator-based sources. This positron accumulator uses a Penning-style trap to collect moderated positrons from a radioactive source. The positron pulses may be emitted with repetition rates in the range of $50-1000 \mathrm{~Hz}$, which is appropriate for $\overrightarrow{\mathrm{H}}$ production schemes involving laser-induced recombination. Bunching techniques may be used to vary the width of the positron pulses over the range 30-120 ns (FWHM) to match the width of the antiproton and/or laser pulses. The efficiency of the accumulator increases from $\sim 10 \%$ at $100 \mathrm{~Hz}$ to $\sim 50 \%$ at $1000 \mathrm{~Hz}$. At $250 \mathrm{~Hz}$ the efficiency is $\sim 25 \%$ and the accumulator has delivered up to $8 \mathrm{e}^{+} /$pulse per $\mathrm{mCi}$ of positron activity. This translates into $\sim 1.2 \times 10^{5} \mathrm{e}^{+} /$pulse for a $100 \mathrm{Ci}^{58}$ Co source.
\end{abstract}

\section{Introduction}

Antihydrogen $(\overline{\mathrm{H}})$ promises to provide a laboratory for many fundamental experiments including sensitive tests of the CPT theorem and investigations of the gravitational properties of antimatter. It may also be useful to probe interactions between antimatter and matter. Furthermore, antihydrogen could provide a source of polarized antiprotons $(\overline{\mathrm{p}})$ for spin-sensitive high-energy experiments.

Several techniques for creating $\overline{\mathrm{H}}$ have been discussed including collisions between $\bar{p}$ and positronium atoms (both in the ground state $[1,2]$ and in excited states [3]) and the use of trapped $\overline{\mathrm{p}} / \mathrm{e}^{+}$plasmas [4]. The Michigan Positron Group has been involved in developing an $\overline{\mathrm{H}}$ formation scheme which employs merged, velocity-matched beams of $\overline{\mathrm{p}}$ and $\mathrm{e}^{+}[5,6]$.

If their velocities are well matched, the co-propagating positrons and antiprotons in the merged beam are essentially antihydrogen "atoms" with energies in a narrow band near the lower edge of the continuum. These "atoms" may undergo spontaneous radiative decay into an $\overline{\mathrm{H}}$ bound state,

$$
\overline{\mathrm{p}}+\mathrm{e}^{+} \rightarrow \overline{\mathrm{H}}+h \nu .
$$

The rate of $\overline{\mathrm{H}}$ formation can be enhanced by roughly two orders of magnitude using laser-induced stimulated recombination $[7,8]$, 


$$
\overline{\mathrm{p}}+\mathrm{e}^{+}+n h \nu \rightarrow \overline{\mathrm{H}}+(n+1) h \nu,
$$

a process which has been experimentally tested with hydrogen $[9,10]$.

The power needed to stimulate recombination into low $n$ states $(n \approx 2-5)$ is high enough that a pulsed laser is required [7]. This suggests the use of a pulsed $\mathrm{e}^{+}$ beam which is synchronized to the $\bar{p}$ and laser beams. Such pulsed $\mathrm{e}^{+}$beams are readily available from accelerators [11,12], but a more convenient and economical source of positrons is provided by $\beta$-emitting radioisotopes.

In order to produce a pulsed $\mathrm{e}^{+}$beam from a radioactive source it is necessary to trap and accumulate the positrons. Other $\mathrm{e}^{+}$accumulators have been reported, but they were operated at repetition rates which were either fairly high $(\approx 1 \mathrm{kHz})$ [13] or very low $(\leqslant 1 \mathrm{~Hz})[14]$ when compared to pulsed lasers.

Presented below are results from the Michigan positron accumulator. The construction of this accumulator was based upon a design which is well suited to $\bar{H}$ production [15]. These results represent a significant improvement over the preliminary results reported previously [16] and the accumulator is presently performing at a level which is useful for many experiments [17]. Below, extrapolations are made to expected $\overline{\mathbf{H}}$ formation rates. Since they are based upon a working instrument, these projections are less speculative than those made previously $[5,15]$.

\section{Accumulator design and performance}

The Michigan positron accumulator relies upon a Penning-style trap in which a uniform magnetic field provides radial confinement while electric fields provide longitudinal confinement. The accumulator is shown schematically in fig. 1 along with the electric potentials applied to the various elements.

Beta-decay positrons emitted from a radioactive source are thermalized within the annealed tungsten vanes of the venetian-blind-style moderator [18]. Slow positrons are re-emitted from the moderator vanes into the trap with a kinetic energy given by

$$
E_{\mathrm{K}}=e\left[V_{\mathbf{M}}(t)-V_{\mathrm{T}}\right]-\phi_{+},
$$

where $V_{\mathrm{M}}(t)$ and $V_{\mathrm{T}}$ are the voltages applied to the moderator and the trap respectively (see fig. 1) and $\phi_{+} \approx-2.8 \mathrm{eV}$ is the workfunction for positrons in tungsten. The efficiency of this moderation process is $\sim(1-3) \times 10^{-4}$.

The moderated positrons traverse the trap and are reflected back toward the source by the potential applied to the gate at the far end $\left(V_{\mathrm{G}}(t)\right.$ in fig. 1). During this transit time $V_{M}(t)$ is constantly rising until the positrons are effectively trapped because they are no longer energetic enough to return to the moderator. At the end of the desired accumulation period $V_{\mathrm{G}}(t)$ is quickly dropped and a pulse of positrons is allowed to leave the trap. The accumulation period can be easily altered 

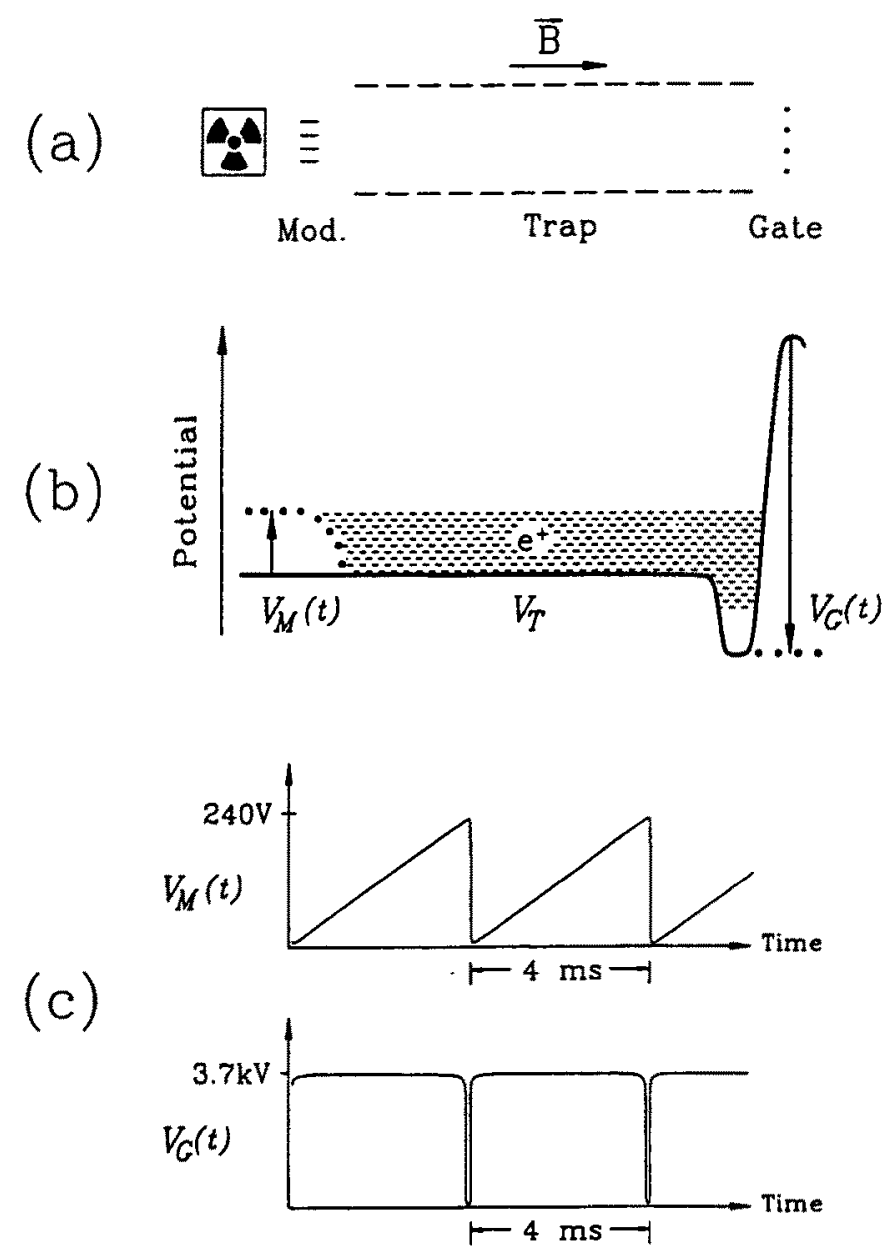

Fig. 1. The configuration of the accumulator is shown schematically in (a). The voltages applied to the accumulator elements are shown in (b) with arrows denoting time-varying voltages. The explicit time dependence of these voltages is shown in (c).

over a range consistent with available voltage ramps and pulsers and is generally determined by the other pulsed beams in the experiment.

In previous discussions of this accumulator it was assumed that the time available to trap the positrons was limited to the duration of a single round-trip $[15,16]$. In this case $V_{M}(t)$ must be raised by $\sim 3 \mathrm{~V}$ (see eq. (3)) during the transit time of positrons in the trap ( $100 \mathrm{~ns})$. This would entail ramps with infeasibly high amplitudes or undesirably high repetition rates [16].

Fortunately, the required $3 \mathrm{~V}$ increase may be applied over many passes for the following reason. When the positrons are reflected by the gate they encounter radial components of electric field. These radial components cause the $\mathrm{e}^{+}$cyclotron orbits to precess about the magnetic field axis (magnetron motion). Thus, because 
the moderator structure has a large open-area, it is possible for positrons to re-enter the region of the moderator without striking the tungsten vanes. As a result, ramps with modest amplitudes and low repetition rates can be used to trap the positrons with high efficiency.

The trap cylinder is broken into many separate sections which may be biased independently. This is done in order to control the shape of the trap potential and study its effect upon the positron pulses. In particular, a small potential well has been placed next to the gate. Its purpose is to further confine any positrons which happen to lose "axial energy" while in the trap (e.g., through scattering from residual gas atoms).

Furthermore, the sectioned trap makes it possible to forcibly eject the positrons from the trap in order to reduce their time spread. A sloped potential is applied to the trap before the gate is lowered. The resultant electric field accelerates positrons near the source for a longer time than it does those near the gate. As a result the positrons arrive on target (e.g., a remoderator) at approximately the same time [13].

A sample output pulse is shown in fig. 2. The pulsewidth is $\sim 30$ ns (FWHM) with the accumulator running at $250 \mathrm{~Hz}$. Both the width and the repetition rate can be easily adjusted, however other modes of operation have not been fully investigated.

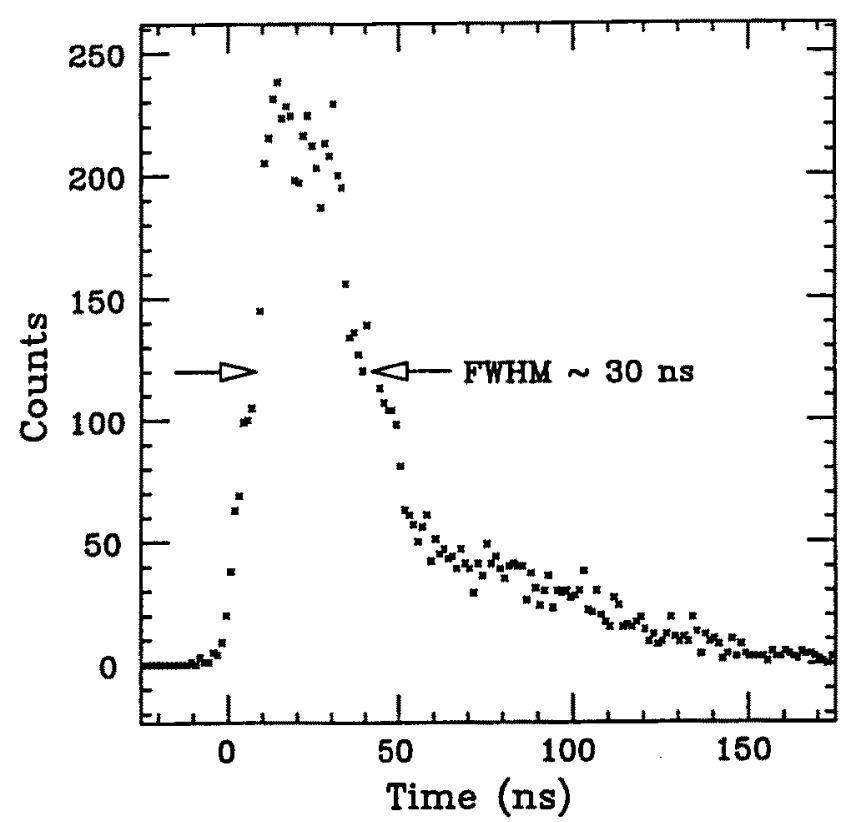

Fig. 2. This is a time spectrum showing the temporal shape of the positron pulses. It represents a sum of $50 \mathrm{k}$ pulses with about 200 positrons each. Note that the positron density is approximately uniform across the $30 \mathrm{~ns}$ pulsewidth. 


\section{Projected antihydrogen formation rates}

The stimulated recombination rate of $\overline{\mathrm{H}}$ atoms can be expressed as

$$
R_{\overline{\mathrm{H}}}=G \alpha n_{\overline{\mathrm{p}}} n_{\mathrm{e}^{+}} V \eta,
$$

where $G$ is the gain factor for stimulated recombination, $\alpha$ is the recombination rate coefficient, $n_{\overline{\mathrm{p}}}\left(n_{\mathrm{e}^{+}}\right)$is the antiproton (positron) density, $V$ is the volume in which the $\overline{\mathrm{p}}$ and $\mathrm{e}^{+}$beams overlap, and $\eta$ is the duty factor for the laser and/or $\mathrm{e}^{+}$ beams.

Calculations for a pulsed laser driving transitions to the $n=2$ level yield $G \approx 100$ [7]. The recombination coefficient has been calculated to be $\alpha=2.2 \times 10^{-12} \mathrm{~cm}^{3} \mathrm{~s}^{-1}$ which is consistent with experimental results from electron cooling experiments with protons [7].

Pulses of antiprotons may be loaded into LEAR until the ring is essentially uniformly populated. These $\overline{\mathrm{p}}$ may then be stored at any velocity from $\beta=0.1$ to $\beta=0.9$. Previous considerations of merged beam formation have assumed velocities of $\beta=0.3$ for both beams [ 6 . The maximum number of $\bar{p}$ which may be stored at this velocity is $\sim 3 \times 10^{9}$ [6]. Since the diameter of the $\overline{\mathrm{p}}$ beam is expected to be $\sim 2 \mathrm{~mm}$ [19], this corresponds to a $\overrightarrow{\mathrm{p}}$ density of $n_{\overline{\mathrm{p}}} \approx 1.3 \times 10^{7} \mathrm{~cm}^{-3}$ for the $75 \mathrm{~m}$ circumference of LEAR.

In order to estimate $n_{\mathrm{e}^{+}}$it is necessary to know the number of positrons per pulse as well as the diameter and velocity of the $\mathrm{e}^{+}$beam. At present, the shortest pulses produced by the accumulator have a FWHM of $\sim 30$ ns. With a $\sim 30 \mathrm{mCi}^{22} \mathrm{Na}$ source, these pulses contain $\sim 100 \mathrm{e}^{+}$within the $30 \mathrm{~ns}$ width $\left(\sim 200 \mathrm{e}^{+}\right.$in the entire pulse). This translates into $\sim 6 \times 10^{4} \mathrm{e}^{+}$per $30 \mathrm{~ns}$ pulse for a source containing 15 Ci of positron activity $\left(17 \mathrm{Ci}\right.$ of ${ }^{22} \mathrm{Na}$ or $100 \mathrm{Ci}$ of ${ }^{58} \mathrm{Co}$ ). Methods of producing such intense sources are currently under investigation at Michigan [20].

The diameter of the $\mathrm{e}^{+}$beam is currently $\sim 4 \mathrm{~mm}$ and the energy spread of the positrons in the pulse is $\sim 400 \mathrm{eV}$. In order to reduce this spread it is necessary to remoderate the positrons. Through remoderation the beam diameter may be reduced by up to an order of magnitude and the energy spread becomes thermal. Although $\sim 70 \%$ of the positrons are lost in this process the density of positrons may be greatly increased [21].

Assuming the velocity and diameter of the $\mathrm{e}^{+}$beam are matched to the $\overline{\mathrm{p}}$ beam, the $30 \mathrm{~ns}$ pulsewidth implies an overlap volume of $V \approx 8 \mathrm{~cm}^{3}$ (in the $\overline{\mathrm{p}}$ rest frame). Hence, the positron density is $n_{\mathrm{e}^{+}} \approx 2.3 \times 10^{3} \mathrm{~cm}^{-3}$.

It is assumed that the laser pulses will have the same width as the positron pulses and that the laser will operate at the repetition rate of the accumulator. For $30 \mathrm{~ns}$ pulses at $250 \mathrm{~Hz}$ the duty factor is only $\eta=7.5 \times 10^{-6}$.

Combining all of these factors via eq. (4) yields a formation rate of $R_{\overline{\mathrm{H}}} \approx 4 \times 10^{-4} \mathrm{~s}^{-1}$ ( $\sim 30$ per day). This rate, though large enough to detect $\overline{\mathrm{H}}$ atoms, is too small for any precise spectroscopic measurements. 
The major obstacle is the low duty factor of the pulsed beams. Schemes have been proposed to recirculate the positrons to improve this factor [15,22]. In particular, consider a positron trap within the LEAR beamline. It should be straightforward to keep a positron pulse trapped in the $\bar{p}$ beam until the next pulse is emitted from the accumulator. This would increase the duty factor of the $\mathrm{e}^{+}$beam to 0.5 since the positron pulse would be counterpropagating (and thus not velocity matched) half of the time. Employing this technique without a laser (i.e. $G=1$ ) yields $R_{\overline{\mathrm{H}}} \approx 0.3 \mathrm{~s}^{-1}$ ( $\sim 15$ per minute). Furthermore, the prospects for significantly increasing $n_{\mathrm{e}^{+}}$in such a trap appear excellent.

However, the duty factor of the laser may also be improved. It should be possible to stimulate transitions to high $n$ states $(n \geqslant 12)$ using a $\mathrm{CO}_{2}$ laser operating in cw mode and still obtain a gain of $G \approx 100$ [8]. Assuming no change in the other parameters of eq. (4) (including $n_{\mathrm{e}^{+}}-\mathrm{a}$ very conservative assumption) the formation rate becomes $R_{\overline{\mathrm{H}}} \approx 30 \mathrm{~s}^{-1}$, which is sufficient for spectroscopy experiments [23].

One might comment that the departure from pulsed beams discussed above calls into question the need for an accumulator. At first glance it seems that the LEAR $\mathrm{e}^{+}$trap could be filled with a standard slow positron beam. It should be noted, however, that the LEAR trap is quite different from an accumulator. In an accumulator one must load positrons into the trap without allowing those already in the trap to escape. Since no increase in $n_{\mathrm{e}^{+}}$is assumed above, such care is not necessary in the LEAR trap (a significant simplification) and an accumulator is still important for providing high positron densities. Lastly, while only the merged beam scheme has been discussed here, the accumulator also has the potential to quickly load plasma traps in schemes employing thermal trapped antiprotons.

\section{References}

[1] B.I. Deutch, L.H. Andersen, P. Hvelplund, F.M. Jacobsen, H. Knudsen, M.H. Holzscheiter, M. Charlton and G. Laricchia, Hyp. Int. 44 (1988) 271.

[2] S.N. Nahar and J.M. Wadehra, Phys. Rev. A 37 (1988) 4118.

[3] M. Charlton, Phys. Lett. Al43 (1990) 143.

[4] G. Gabrielse, S.L. Rolston, L. Haarsma and W. Kells, Phys. Lett. A129(1988) 38.

[5] A. Rich, R. Conti, W. Frieze, D.W. Gidley, H. Griffin, M. Skalsey, T. Steiger, J. Van House, W. Zheng and P.W. Zitzewitz, in: Atomic Physics with Positrons, eds. J.W. Humberston and E.A.G. Armour (Plenum Press, New York, 1987) p. 321.

[6] H. Poth, B. Seligmann, W. Schwab, M. Wörtge, A. Wolf, R. Conti, W. Frieze, D. Gidley, A. Rich, M. Skalsey, J. Van House, P. Zitzewitz, J. Berger, P. Blatt, R. Neumann and G. zu Putlitz, Hyp. Int. 44(1988) 259.

[7] R. Neumann, H. Poth, A. Winnacket and A. Wolf, Z. Phys. A 313 (1983) 253.

[8] P. Blatt, Hyp. Int. 44 (1988) 295.

[9] U. Schramm, J. Berger, M. Grieser, D. Habs, E. Jaeschke, G. Kilgus, D. Schwalm, A. Wolf, R. Neumann and R. Schuch, Phys. Rev. Lett. 67 (1991) 22. 
[10] F.B. Yousif, P. Van der Donk, Z. Kucherovsky, J. Reis, E. Brannen, J.B.A. Mitchell and T.J. Morgan, Phys. Rev. Lett. 67 (1991) 26.

[11] L.D. Hulett Jr., T.A. Lewis, R.G. Alsmiller Jr., R. Peelle, S. Pendyala, J.M. Dale and T.M. Rosseel, Nucl. Instr. Meth. B24/25 (1987) 905.

[12] A.P. Mills Jr., E.D. Shaw, R.J. Chichester and D.M. Zuckerman, Rev. Sci. Instr. 60 (1989) 825.

[13] A.P. Mills Jr., in: Positron Scattering in Gases, eds. J.W. Humberston and M.R.C. McDowell (Plenum Press, New York, 1984) p. 121.

[14] C.M. Surko, M. Leventhal and A. Passner, Phys. Rev. Lett. 62 (1989) 901.

[15] R. Conti, A. Rich, D. Gidley, M. Skalsey, J. Van House, P.W. Zitzewitz, H. Poth, W. Schwab, B. Seligmann, M. Wörtge and A. Wolf, Hyp. Int. 44 (1988) 201.

[16] R.S. Conti, B. Ghaffari and T.D. Steiger, Nucl. Instr. Meth. A299(1990) 420.

[17] T.D. Steiger, B. Ghaffari, A. Rich and R.S. Conti, Abstracts of Contributed Papers 12th Int. Conf. on Atomic Physics, Ann Arbor 1990, eds. W.E. Baylis, G.W.F. Drake and J.M. McConkey (University of Windsor, Windsor, 1990) p. I-22.

[18] J. Van House and P.W. Zitzewitz, Phys. Rev. A 29 (1984) 96.

[19] H. Poth, C. Habfast, W. Schwab, B. Seligmann, M. Wörtge, A. Wolf, H. Haseroth, C.E. Hill and J.L. Vallet, in: Physics at LEAR with Low Energy Antiprotons, eds. C. Amsler, G. Backenstoss, R. Klapisch, C. Leluc, D. Simon and L. Tauscher (Harwood, Chur, 1988) p. 121.

[20] T.D. Steiger, J. Stehr, H.C. Griffin, J.H. Rogers, M. Skalsey and J. Van House, Nucl. Instr. Meth. A299 (1990) 255.

[21] P.J. Schultz, E.M. Gullikson and A.P. Mills Jr., Phys. Rev. B 34 (1986) 442.

[22] A.S. Artamanov, Ya.S. Derbenev and E.L. Saldin, Part. Accel. 23 (1988) 79.

[23] R. Neumann, Hyp. Int. 44(1988) 305. 